\title{
Genetic variations in the IGF-IGFR-IGFBP axis confer susceptibility to lung and esophageal cancer
}

\author{
X.P. Huang, W.H. Zhou and Y.F. Zhang \\ Department of Cardiothoracic Surgery, Second Affiliated Hospital, \\ Wenzhou Medical College, Wenzhou, China \\ Corresponding author: X.P. Huang \\ E-mail: wz2h_hxp@126.com
}

Genet. Mol. Res. (2014) Ahead of Print

Received June 12, 2013

Accepted November 29, 2013

Published January 24, 2014

DOI http://dx.doi.org/10.4238/2014.January.24.17

\begin{abstract}
Recent evidence suggests that genetic variations in the insulin-like growth factor (IGF)-IGF receptor (IGFR)-IGF binding proteins (IGFBP) axis may impact an individual's susceptibility to lung and esophageal cancer, but individually published results are inconclusive. Our meta-analysis aimed at providing a more precise estimation of these associations. An extensive literature search was conducted for appropriate articles published before May 15th, 2013. This meta-analysis was performed using the STATA 12.0 software. The crude odds ratios (ORs) with $95 \%$ confidence intervals (CIs) were calculated for each study and then pooled using a random effect model. Twelve case-control studies were included with a total of 2686 lung cancer patients, 771 esophageal cancer patients, and 5918 healthy controls. Our meta-analysis indicated that genetic variations in the IGF-IGFR-IGFBP axis may be associated with increased risk of lung and esophageal cancer, especially among Asian populations. Further subgroup analysis by gene type indicated that common polymorphisms in the IGF1/2, IGF-1R, and IGFBP-3/5 genes may be the main determinants for lung cancer risk, while IGF-1, IGF-
\end{abstract}


X.P. Huang et al.

$1 \mathrm{R}$, and IGFBP-1 genetic polymorphisms may increase the risk of esophageal cancer. The current meta-analysis suggests that genetic variations in the IGF-IGFR-IGFBP axis confer susceptibility to lung and esophageal cancer, especially among Asian populations. Common polymorphisms in the IGF-IGFR-IGFBP axis may serve as useful biomarkers for predicting the risk of lung and esophageal cancer.

Key words: Lung cancer; Esophageal cancer; Polymorphism; Insulin-like growth factor; Meta-analysis

\section{INTRODUCTION}

Cancer remains an important public health problem in both developed and developing countries (Braithwaite et al., 2012). In 2008, an estimated 7.6 million people died of cancer worldwide, accounting for $13 \%$ of the global mortality (Jemal et al., 2011). It is generally recognized that cancer is a multifactorial disease caused by complex interactions between environmental and genetic factors (Ponder, 2001). However, the exact cellular and molecular mechanisms leading to the development of cancer remain unclear. Recently, a large number of candidate genes responsible for the genesis of various cancers have been identified (Vogelstein and Kinzler, 2004). Determination of single nucleotide polymorphisms (SNPs) in candidate genes may prove reliable in predicting the genetic risk of cancer, and might thus contribute to the primary prevention of this condition (Tabor et al., 2002).

The insulin-like growth factor (IGF) signaling pathway plays an important role in regulating cellular proliferation and apoptosis (Pollak et al., 2004). The biological activities of the IGFs are modulated by IGF-binding proteins (IGFBPs) and mediated by IGF receptors (IGFRs) (Yi et al., 2005). Emerging interest in the IGF-IGFR-IGFBP axis and its effect on carcinogenesis has increased recently because high IGF-1 serum concentrations were associated with an increased risk of lung, gastric, esophageal, breast, prostate, and colorectal cancers (Furstenberger and Senn, 2002). Therefore, it was hypothesized that genetic variations in the IGF-IGFR-IGFBP axis might be associated with cancer risk. A recent meta-analysis of 17 case-control studies by Chen et al. (2008) assessed the association between the IGF-1 (CA)19 repeat polymorphism and the risk of prostate, breast, and colorectal cancers. Their results indicated that the IGF-1 (CA)19 polymorphism may not be a major determinant of susceptibility to cancer. Because this previous meta-analysis did not provide convincing and reliable evidence in associating the IGF-IGFRIGFBP axis to the risk of cancer risk, we performed a meta-analysis of published data to provide a more comprehensive and reliable conclusion on the associations between genetic variations in the IGF-IGFR-IGFBP axis and susceptibility to lung and esophageal cancer.

\section{MATERIAL AND METHODS}

\section{Search strategy and selection criteria}

An extensive literature search for relevant studies was conducted in the PubMed, Embase, Web of Science, Cochrane Library, and CBM databases from inception through May 15th, 2013. 
IGF-IGFR-IGFBP axis and cancer risk

We used the following keywords and MeSH terms: "lung cancer", "esophageal cancer", "IGF", "IGFR", "IGFBP", and "polymorphism". There was no language restriction. Manual searches of reference lists from potentially relevant articles were also performed to identify other potential studies. To be included in the analysis, these studies had to meet the following criteria: (1) case-control studies focusing on the associations between genetic variations in the IGF-IGFR-IGFBP axis and susceptibility to lung or esophageal cancer; (2) all patients diagnosed with lung or esophageal cancer were confirmed by histopathological examinations; (3) published data about genotype frequencies of polymorphisms was sufficient; (4) the genotype distribution in healthy controls conformed to Hardy-Weinberg equilibrium (HWE). Studies were excluded if they did not meet all of these inclusion criteria. Any disagreements were resolved by discussions and subsequent consensus.

\section{Data extraction}

Two authors independently extracted data from eligible studies by using a standardized form. The following information was collected prospectively: surname of first author, year of publication, source of publication, country of origin, ethnicity, language of publication, study type, total number of subjects, source of cases and controls, pathological type, gene type, DNA sample, SNP detection method, genotype frequencies, and evidence of HWE in controls. In cases of conflicting evaluations, disagreements of inconsistent data from the eligible studies were resolved through discussions and careful reexaminations of the full text by the authors.

\section{Quality assessment}

The quality of included studies was assessed independently by two authors (Zhou and Zhang) based on the STROBE quality score systems (da Costa et al., 2011). Forty assessment items related to quality appraisal were used in this meta-analysis with scores ranging from 0 to 40 . The included studies were classified into three levels based on their scores: low quality (0-19), moderate quality (20-29), and high quality (30-40), respectively. Disagreements of STROBE scores of the included studies were resolved through a comprehensive reassessment by the authors.

\section{Statistical analysis}

The odds ratios (ORs) and 95\% confidence intervals (CIs) were calculated under five genetic models: allele model (mutant [M] allele versus wild [W] allele), dominant model (WM + MM versus WW), recessive model (MM versus WW + WM), homozygous model (MM versus WW), and heterozygous model (MM versus WM). The significance of the pooled estimate was determined using the $Z$ test. Genotype distributions in the control subjects were tested for HWE by the chi-squared test. We estimated the degree of heterogeneity among studies using Cochran's $Q$-statistic, which was considered significant at $\mathrm{P}<0.05$ (Jackson et al., 2012). The $P^{2}$ test was also used to quantify the heterogeneity (ranging from 0 to 100\%) (Peters et al., 2006). When a significant $Q$-test with $\mathrm{P}<0.05$ or $P^{2}>50 \%$ indicated heterogeneity among studies, the random effects model (DerSimonian Laird method) was applied to the meta-analysis; otherwise, the fixed effects model (Mantel-Haenszel method) was used. In order to explore sources of heterogeneity, subgroup analyses were performed based on ethnicity, gene type, source of control, and SNP detection method. To evaluate the influence of individual studies on the overall risk estimate, we conducted a sensitivity analysis by omitting each study in turn. Funnel plots and Egger's linear regression test were used 
X.P. Huang et al.

to assess the potential publication bias of included studies (Zintzaras and Ioannidis, 2005). All tests were two-sided, and a $\mathrm{P}$ value of $<0.05$ was considered statistically significant. All analyses were conducted using STATA software, version 12.0 (Stata Corp; College Station, TX, USA).

\section{RESULTS}

\section{Baseline characteristics of studies included}

A total of 161 articles relevant to the searched keywords were initially identified. Of these articles, 82 were excluded after reviewing titles and key words. After reviewing the abstracts and full texts, another 65 papers were excluded. Finally, 12 case-control studies met our inclusion criteria (Moon et al., 2006; Rudd et al., 2006; Han et al., 2008; Jia et al., 2008; Kotsinas et al., 2008; Hoyo et al., 2009; Jia et al., 2009; MacDonald et al., 2009; McElholm et al., 2010; Dong et al., 2011; Li et al., 2012; Lin et al., 2012). The publication years of the involved studies ranged from 2006 to 2012. A flow chart of the selection procedures is shown in Figure 1. A total of 9375 subjects were involved in this meta-analysis, including 2686 lung cancer patients, 771 esophageal cancer patients, and 5918 healthy controls. Overall, there were six lung cancer studies and six esophageal cancer studies. Six studies used hospital-based controls, while the other six studies used population-based controls (community populations). Seven studies were conducted in Asian populations and five studies were conducted in Caucasian populations. The DNA samples used for examination of genetic polymorphisms were extracted from blood in all included studies. Genotype methods included polymerase chain reaction-restriction fragment length polymorphism (PCR-RELP), direct DNA sequencing, TaqMan assay, and the high resolution melting method. Seven genes in the IGF-IGFRIGFBP axis were addressed, including the IGF-1, IGF-2, IGF-1R, IGF-2R, IGFBP1, IGFBP3, and IGFBP5 genes. HWE tests were conducted on the genotype distribution of the controls in all twelve studies, and no study deviated from HWE (all P > 0.05). All quality scores of the included studies were higher than 20 (moderate-high quality). The characteristics and methodological quality of the included studies are summarized in Table 1.

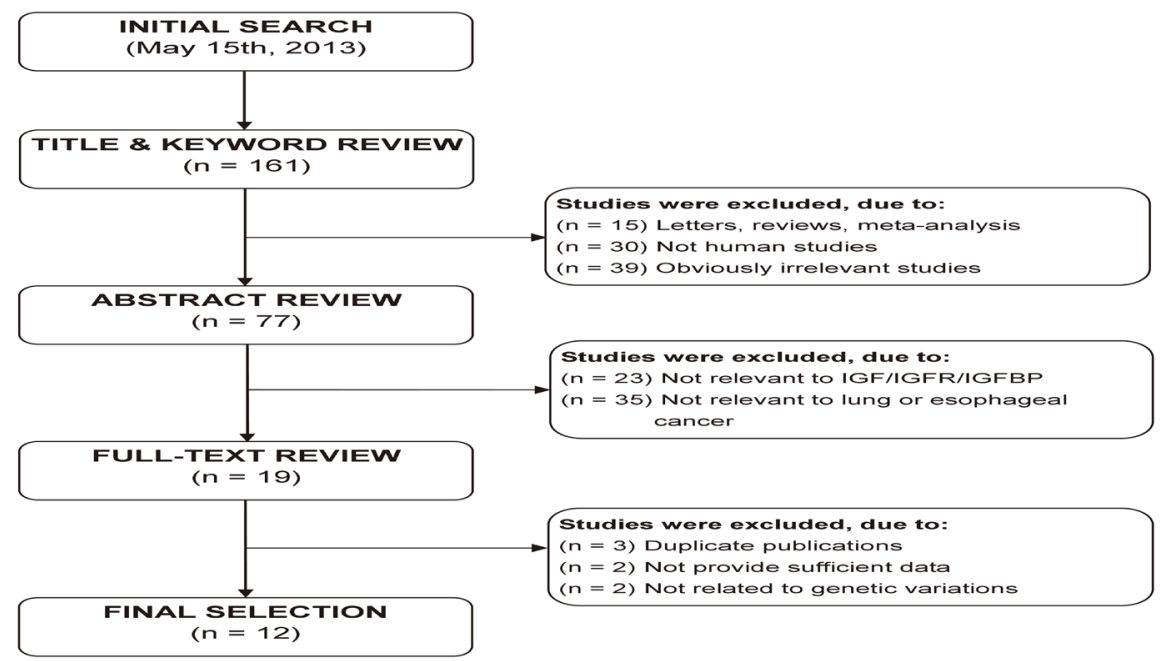

Figure 1. Flow chart of literature search and study selection. 
IGF-IGFR-IGFBP axis and cancer risk

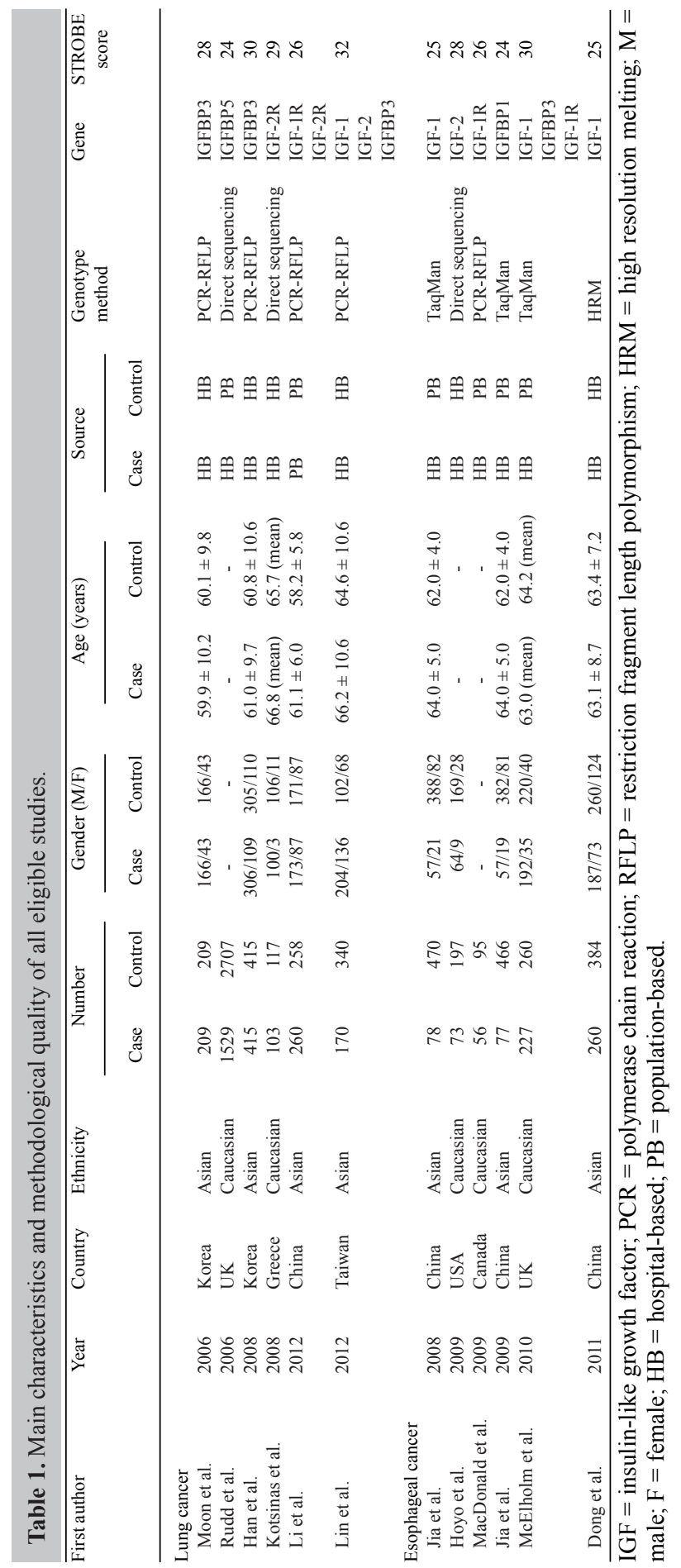


X.P. Huang et al.

\section{Genetic variations in the IGF-IGFR-IGFBP axis and lung cancer risk}

The association between genetic variations in the IGF-IGFR-IGFBP axis and lung cancer risk was evaluated in six studies. The heterogeneity was not significant (all $\mathrm{P}>0.05$ ), and therefore the fixed effects model was used. The meta-analysis results showed that genetic variations in the IGF-IGFR-IGFBP axis could increase the risk of lung cancer (allele model: $\mathrm{OR}=1.37,95 \% \mathrm{CI}=1.23-1.52, \mathrm{P}<0.001$; dominant model: $\mathrm{OR}=2.01,95 \% \mathrm{CI}=1.24-3.27$, $\mathrm{P}=0.005$; recessive model: $\mathrm{OR}=1.24,95 \% \mathrm{CI}=1.11-1.38, \mathrm{P}<0.001$; homozygous model: $\mathrm{OR}=2.14,95 \% \mathrm{CI}=1.57-2.92, \mathrm{P}<0.001)($ Figure 2$)$. However, no significant associations were observed under the heterozygous models $(\mathrm{OR}=1.05,95 \% \mathrm{CI}=0.87-1.28, \mathrm{P}=0.609)$. Subgroup analysis by gene type indicated that the IGF1/2, IGF-1R, and IGFBP $3 / 5$ genes may be the main determinants of lung cancer risk, but the IGF-2R gene did not show any association with increased risk of lung cancer. Further subgroup analyses indicated that there were significant associations between genetic variations in the IGF-IGFR-IGFBP axis and lung cancer risk in Asian populations, population-based, hospital-based, and PCR-RFLP studies (as shown in Table 2). However, no statistically significant association was found in Caucasian populations and in non-PCR-RFLP studies (all $\mathrm{P}>0.05$ ).

\begin{tabular}{|c|c|c|c|}
\hline Study ID & $\begin{array}{c}\text { Allele model } \\
\text { (M allele versus } W \text { allele) }\end{array}$ & OR (95\% CI) & Weight \% \\
\hline Moon JW (2006) & & $1.57(1.14,2.16)$ & 9.15 \\
\hline Rudd MF (2006) & & $1.32(1.06,1.64)$ & 16.00 \\
\hline Han SG (2008) & & $1.24(1.06,1.45)$ & 23.91 \\
\hline Kotsinas A (2008) & & $1.05(0.71,1.55)$ & 6.68 \\
\hline Li HR (2012) & & $1.64(1.37,1.97)$ & 20.31 \\
\hline Lin $\mathrm{IH}(2012)$ & & $1.34(1.15,1.57)$ & 23.95 \\
\hline Overall $\left(I^{2}=36.1 \%, P=0.166\right)$ & & $1.37(1.23,1.52)$ & 100.00 \\
\hline NOTE: Fixed effects model & & & \\
\hline 0.462 & 1 & 16 & \\
\hline Study ID & $\underset{(W M+M M \text { versus } W W)}{\text { Dominant model }}$ & OR (95\% CI) & Weight \% \\
\hline Moon JW (2006) & I & $2.71(0.95,7.73)$ & 11.25 \\
\hline Rudd MF (2006) & & $1.13(0.28,4.53)$ & 8.08 \\
\hline Han SG (2008) & & $2.38(1.67,3.41)$ & 20.98 \\
\hline Kotsinas A (2008) & & $0.93(0.50,1.72)$ & 17.18 \\
\hline Li HR (2012) & & $4.46(3.05,6.52)$ & 20.69 \\
\hline Lin IH (2012) & & $1.57(1.17,2.10)$ & 21.83 \\
\hline Overall $\left(I^{2}=81.5 \%, P<0.001\right)$ & & $2.01(1.24,3.27)$ & 100.00 \\
\hline NOTE: Random effects model & & & \\
\hline
\end{tabular}

Figure 2. Forest plots for the associations between genetic polymorphisms in the IGF-IGFR-IGFBP axis and lung cancer risk. The squares and horizontal lines correspond to the study specific OR and 95\%CI. The area of the squares reflects the weight (inverse of the variance). The diamond represents the summary OR and $95 \%$ CI. 
IGF-IGFR-IGFBP axis and cancer risk

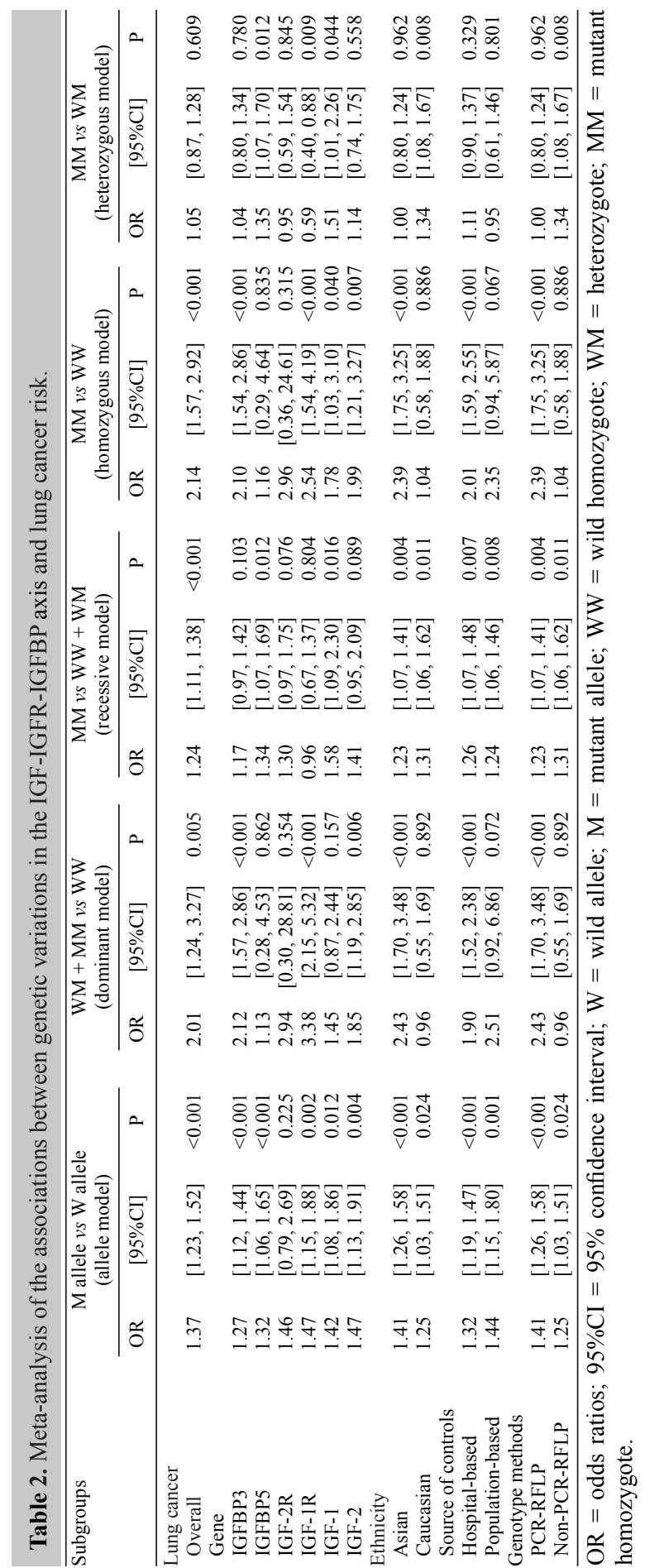


X.P. Huang et al.

\section{Genetic variations in the IGF-IGFR-IGFBP axis and esophageal cancer risk}

Six studies evaluated the association between genetic variations in the IGF-IGFRIGFBP axis and esophageal cancer risk. Since no significant heterogeneity was observed (all $\mathrm{P}>0.05$ ), the fixed effects model was used. Meta-analysis of these studies showed significant associations between genetic variations in the IGF-IGFR-IGFBP axis and increased risk of esophageal cancer (allele model: $\mathrm{OR}=1.26,95 \% \mathrm{CI}=1.08-1.47, \mathrm{P}=0.003$; dominant model: $\mathrm{OR}=1.40,95 \% \mathrm{CI}=1.06-1.86, \mathrm{P}=0.020$ ) (Figure 3 ), but no significant associations were observed under the other genetic models (all $\mathrm{P}>0.05$ ). In the subgroup analysis based on gene type, the results showed that the IGF-1, IGF-1R, and IGFBP1 gene polymorphisms could be associated with increased risk of esophageal cancer, but there was no evidence for any association of the IGF-2, IGF-2R, and IGFBP3 genes. We also performed further subgroup analyses based on ethnicity, source of controls, and SNP detection method. These results suggested significant associations between genetic variations in the IGF-IGFR-IGFBP axis and esophageal cancer risk in Asian populations, hospital-based, and TaqMan subgroups (as shown in Table 3).

\begin{tabular}{|c|c|c|c|}
\hline Study ID & $\begin{array}{c}\text { Allele model } \\
\text { (M allele versus } W \text { allele) }\end{array}$ & OR $(95 \% \mathrm{CI})$ & Weight $\%$ \\
\hline Jia YB (2008) & & \multicolumn{2}{|c|}{$1.06(0.74,1.51) 12.58$} \\
\hline Hoyo C (2009) & & \multicolumn{2}{|c|}{$1.06(0.69,1.61) 9.73$} \\
\hline MacDonald K (2009) & & \multicolumn{2}{|c|}{$0.77(0.48,1.23) 8.14$} \\
\hline Jia YB (2009) & & \multicolumn{2}{|c|}{$1.35(1.06,1.73) 18.96$} \\
\hline McElholm AR (2010) & & \multicolumn{2}{|c|}{$1.37(1.22,1.52) 31.81$} \\
\hline Dong CQ (2011) & & \multicolumn{2}{|c|}{$1.56(1.21,2.00) 18.78$} \\
\hline Overall $\left(I^{2}=48.5 \%, P=0.084\right)$ & & \multicolumn{2}{|c|}{$1.26(1.08,1.47) 100.00$} \\
\hline NOTE: Fixed effects model & & & \\
\hline 0.477 & \multirow[b]{2}{*}{$\begin{array}{l}\text { Dominant model } \\
(W M+M M \text { versus } W W)\end{array}$} & \multicolumn{2}{|l|}{2.1} \\
\hline Study ID & & OR $(95 \% \mathrm{CI})$ & Weight $\%$ \\
\hline Jia YB (2008) & : & \multicolumn{2}{|c|}{$1.21(0.74,1.97) 18.16$} \\
\hline Hoyo C (2009) & & $1.07(0.67,1.71)$ & 19.12 \\
\hline MacDonald K (2009) & 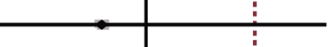 & $0.87(0.44,1.75)$ & 11.72 \\
\hline Jia YB (2009) & : & $1.67(1.17,2.40)$ & 24.37 \\
\hline Dong CQ (2011) & & $1.97(1.43,2.71)$ & 26.62 \\
\hline Overall $\left(I^{2}=51.3 \%, P=0.084\right)$ & & $1.40(1.06,1.86)$ & 100.00 \\
\hline NOTE: Fixed effects model & & & \\
\hline
\end{tabular}

Figure 3. Forest plots for the associations between genetic polymorphisms in the IGF-IGFR-IGFBP axis and esophageal cancer risk. The squares and horizontal lines correspond to the study specific OR and $95 \%$ CI. The area of the squares reflects the weight (inverse of the variance). The diamond represents the summary OR and $95 \%$ CI. 
IGF-IGFR-IGFBP axis and cancer risk

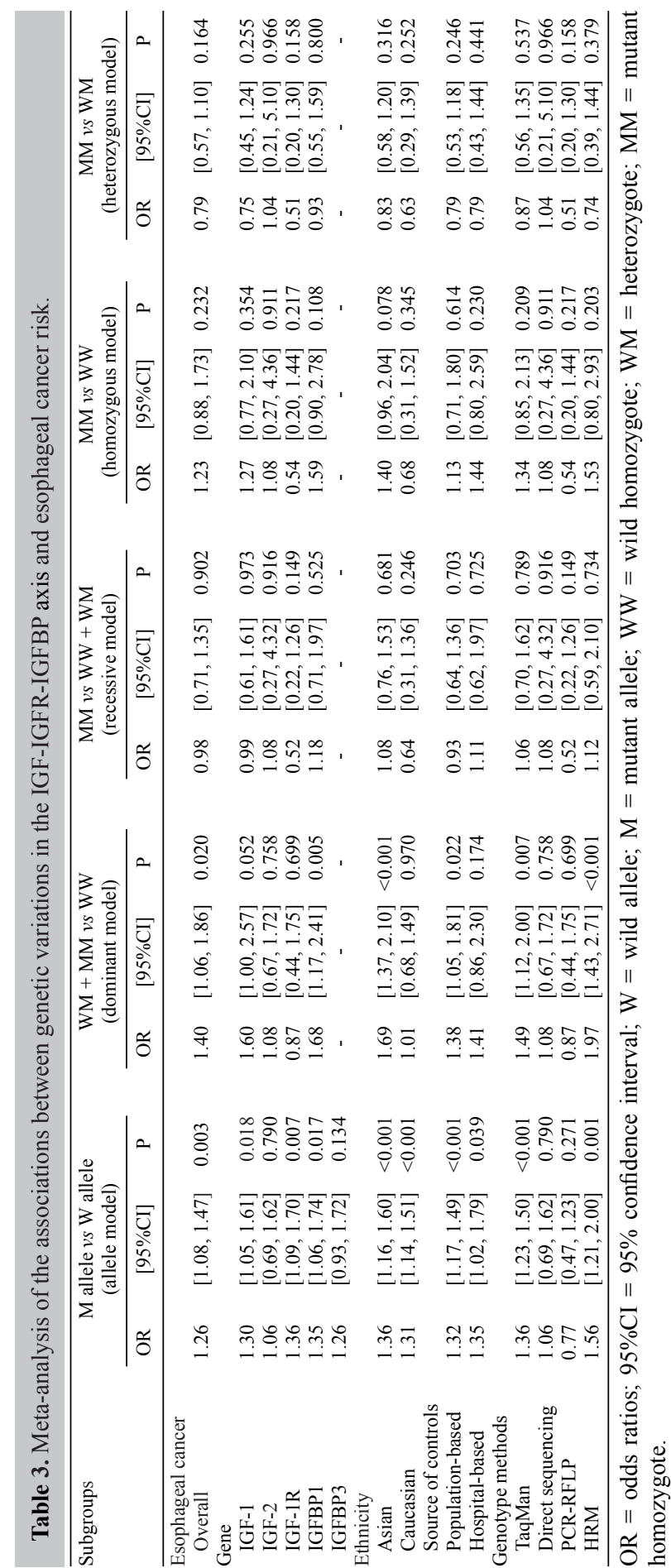


X.P. Huang et al.

\section{Sensitivity analysis and publication bias}

The results of the sensitivity analysis suggested that no individual studies significantly affected the pooled estimates under any of the genetic models (Figure 4). The shapes of the funnel plots did not reveal any evidence of obvious asymmetry (Figure 5). Egger's test also showed that there was no strong statistical evidence of publication bias (all $\mathrm{P}>0.05)$.
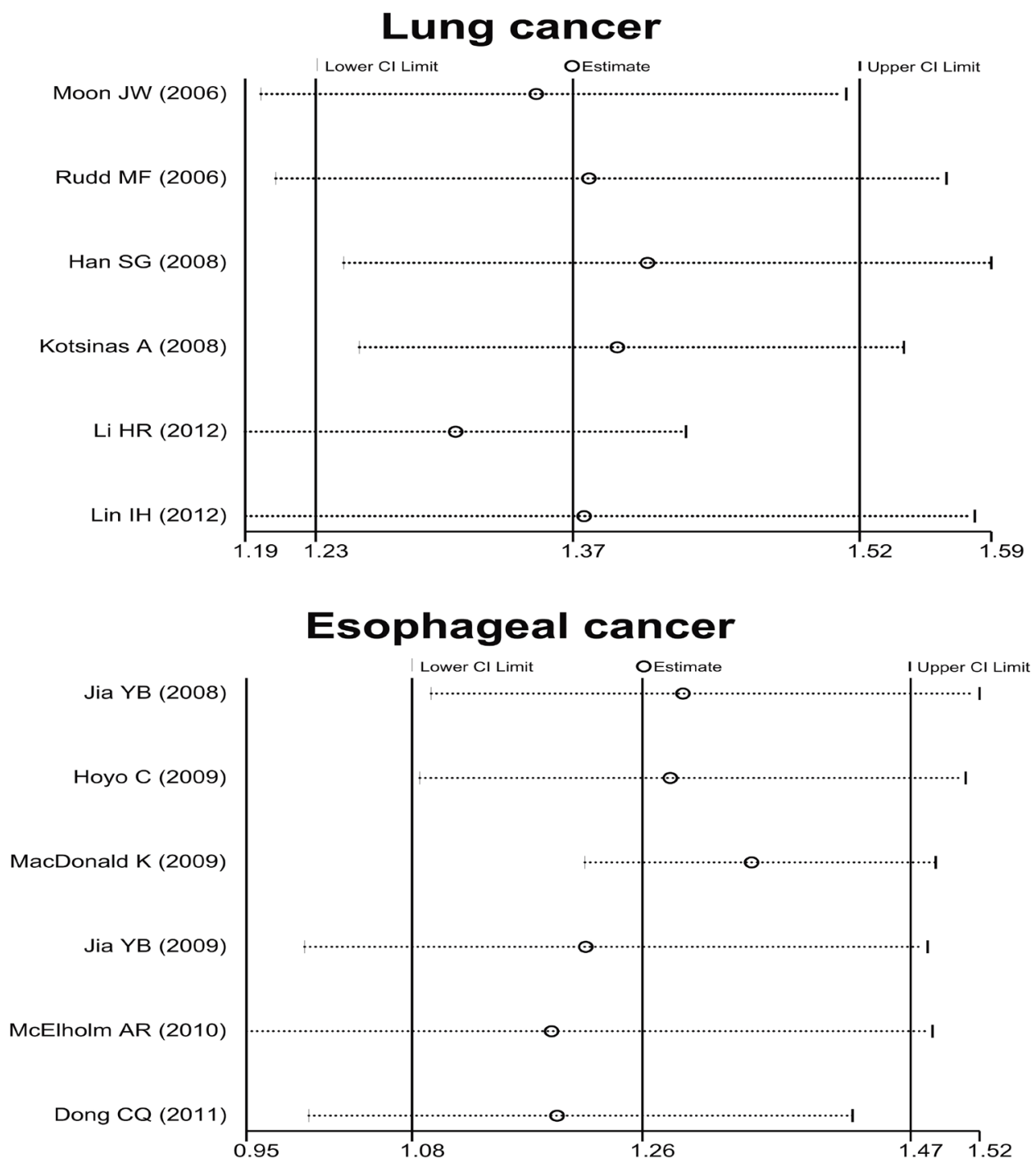

Figure 4. Sensitivity analysis for the associations between genetic polymorphisms in the IGF-IGFR-IGFBP axis and lung and esophageal cancer risk under allele model. Results were computed by omitting each study in turn. Meta-analysis random-effects estimates (exponential form) were used. The two ends of the dotted lines represent the $95 \% \mathrm{CI}$. 
IGF-IGFR-IGFBP axis and cancer risk
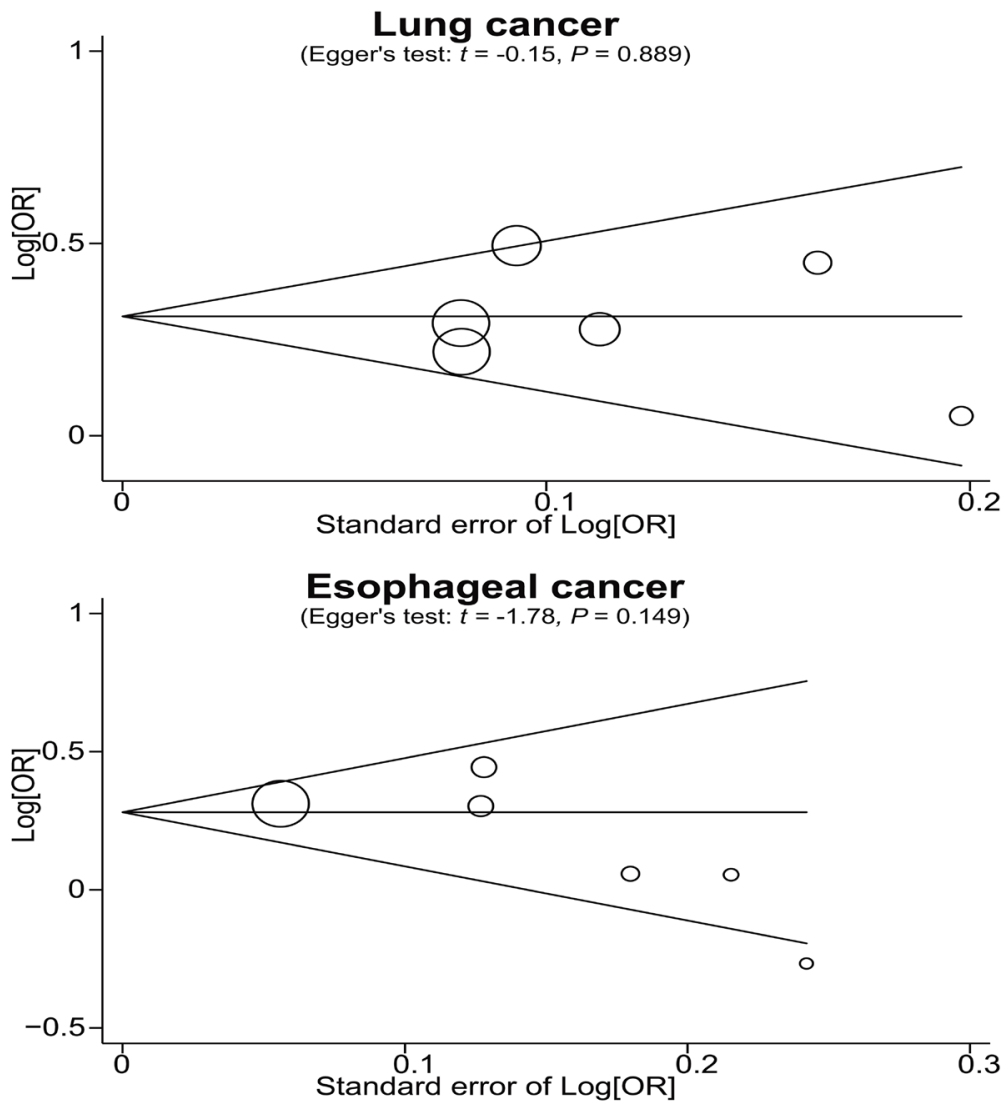

Figure 5. Funnel plots for the associations between genetic polymorphisms in the IGF-IGFR-IGFBP axis and lung and esophageal cancer risk under allele model. Each point represents a separate study for the indicated association. $\log [\mathrm{OR}]$, natural logarithm of OR. Horizontal line, mean magnitude of the effect. Note: Funnel plot with pseudo $95 \%$ confidence limits was used.

\section{DISCUSSION}

IGFs are well known as key regulators of normal carbohydrate and lipid metabolism and growth (Jones and Clemmons, 1995; Kaaks, 2004). The bioactivity of IGFs is modulated by IGFBPs, which have high affinity for both IGF-1 and IGF-2. In general, IGFBPs limit IGFs' access to IGF-1R/2R, thereby attenuating the bioactivity of these growth factors (Pollak, 2008). Several genetic polymorphisms in IGF1/2, IGF-1R/2R, and IGFBP-1 6 (also called the IGF-IGFR-IGFBP axis) have been identified for predicting the development, progression, and clinical outcomes of lung and esophageal cancers (Rudd et al., 2006; Lettre et al., 2007). Many previous genetic studies have suggested that genetic polymorphisms in the IGF-IGFRIGFBP axis may play an important role in lung and esophageal carcinogenesis (Moon et al., 2006; Rudd et al., 2006; Han et al., 2008; Kotsinas et al., 2008; Hoyo et al., 2009; MacDonald et al., 2009; McElholm et al., 2010; Dong et al., 2011; Li et al., 2012; Lin et al., 2012), while other studies found no convincing evidence of these polymorphisms in increasing the risks 


\section{X.P. Huang et al.}

of lung and esophageal cancer (Jia et al., 2008; Jia et al., 2009). This controversy could be explained by several factors, including differences in study designs, sample sizes, ethnicities, statistical methods, etc. Therefore, this meta-analysis aimed to provide a more comprehensive and reliable conclusion of these associations.

This is the first meta-analysis focused on the associations between genetic polymorphisms in the IGF-IGFR-IGFBP axis and the risk of lung and esophageal cancer. In the present meta-analysis, 12 independent case-control studies were included with a total of 2686 lung cancer patients, 771 esophageal cancer patients, and 5918 healthy controls. When all eligible studies were pooled into the meta-analysis, the results showed that common polymorphisms in the IGF-IGFR-IGFBP axis were associated with increased risks of lung and esophageal cancer, especially among Asian populations, while similar associations were not observed among Caucasian populations. Although ethnic differences in susceptibility to lung and esophageal cancer are well known, the molecular basis is not fully understood. One possible reason for these ethnic differences could be that large differences in common SNPs in the IGF-IGFRIGFBP axis that influence the risk of lung and esophageal cancer are mostly due to genetic drift and natural selection (Serre et al., 2008). Further subgroup analysis by gene type indicated that common polymorphisms in the IGF1/2, IGF-1R, and IGFBP-3/5 genes may be the main determinants for lung cancer risk, whereas polymorphisms in the IGF-1, IGF-1R, and IGFBP-1 genes may increase the risk of esophageal cancer. Although no significant association was found for IGF-2R genetic polymorphisms, this result might have lacked sufficient reliability due to small sample size. These findings are consistent with the previous hypothesis that genetic variations in the IGF-IGFR-IGFBP axis may confer susceptibility to lung and esophageal cancer, suggesting that they may be useful as biomarkers for predicting an individual's genetic susceptibility to lung and esophageal cancer.

Our meta-analysis has several limitations that should be acknowledged. The first limitation is that the sample size of this meta-analysis was relatively small and may not have sufficient statistical power in estimating the relationships between the IGF-IGFR-IGFBP axis and lung and esophageal cancer risk. Therefore, more studies with larger sample sizes are still needed. On the other hand, a meta-analysis is a type of a retrospective study and may encounter recall or selection bias, thereby possibly influencing the reliability of our results (Stroup et al., 2000). Most important of all, lack of access to the original study data limited further evaluations of the potential value of these polymorphisms in the IGF-IGFR-IGFBP axis.

In conclusion, our meta-analysis indicates that genetic variations in the IGF-IGFRIGFBP axis confer susceptibility to lung and esophageal cancer, especially among Asian populations. Common polymorphisms in the IGF-IGFR-IGFBP axis may serve as useful biomarkers for predicting the risk of lung and esophageal cancer. Considering the limitations mentioned above, detailed studies are needed to confirm our findings.

\section{REFERENCES}

Braithwaite D, Boffetta P, Rebbeck TR and Meyskens F (2012). Cancer prevention for global health: a report from the ASPO International Cancer Prevention Interest Group. Cancer Epidemiol. Biomarkers Prev. 21: 1606-1610.

Chen X, Guan J, Song Y, Chen P, et al. (2008). IGF-I (CA) repeat polymorphisms and risk of cancer: a meta-analysis. $J$. Hum. Genet. 53: 227-238.

da Costa BR, Cevallos M, Altman DG, Rutjes AW, et al. (2011). Uses and misuses of the STROBE statement: bibliographic study. BMJ Open 1: e000048.

Dong CQ, Liu C, Ma SJ and Gu HY (2011). Association of insulin- like growth factor- 1 polymorphism and risk of 


\section{IGF-IGFR-IGFBP axis and cancer risk}

esophageal cancer. J. Jiangsu Univ. (Medicine Edition) 21: 535-537.

Furstenberger G and Senn HJ (2002). Insulin-like growth factors and cancer. Lancet Oncol. 3: 298-302.

Han SG, Park KH, Sung JS, Whang YM, et al. (2008). Single nucleotide polymorphisms of IGFBP-3 gene and lung cancer risk in a Korean population. Lung Cancer 62: 152-161.

Hoyo C, Schildkraut JM, Murphy SK, Chow WH, et al. (2009). IGF2R polymorphisms and risk of esophageal and gastric adenocarcinomas. Int. J. Cancer 125: 2673-2678.

Jackson D, White IR and Riley RD (2012). Quantifying the impact of between-study heterogeneity in multivariate metaanalyses. Stat. Med. 31: 3805-3820.

Jemal A, Bray F, Center MM, Ferlay J, et al. (2011). Global cancer statistics. CA Cancer J. Clin. 61: 69-90.

Jia YB, Zhang JZ and Yan Y (2008). Association study between a polymorphism in the IGF-I gene and esophageal squamous cell carcinoma. J. Baotou Med. Coll. 24: 452-453.

Jia YB, Dong WJ, Cao Y and Yan Y (2009). No Association Between Two SNPs in the IGFBP1 Gene and Esophageal Squamous Cell Carcinoma. J. Baotou Med. Coll. 25: 7-8.

Jones JI and Clemmons DR (1995). Insulin-like growth factors and their binding proteins: biological actions. Endocr. Rev. 16: 3-34.

Kaaks R (2004). Nutrition, insulin, IGF-1 metabolism and cancer risk: a summary of epidemiological evidence. Novartis Found. Symp. 262: 247-260.

Kotsinas A, Evangelou K, Sideridou M, Kotzamanis G, et al. (2008). The 3' UTR IGF2R-A2/B2 variant is associated with increased tumor growth and advanced stages in non-small cell lung cancer. Cancer Lett. 259: 177-185.

Lettre G, Butler JL, Ardlie KG and Hirschhorn JN (2007). Common genetic variation in eight genes of the GH/IGF1 axis does not contribute to adult height variation. Hum. Genet. 122: 129-139.

Li HR, Chen YS, Shao H, Han LL, et al. (2012). Association between polymorphisms of insulin-like growth factor receptor gene and susceptibility to non-small-cell lung cancer in Fujian Chinese. Zhonghua Yi Xue Yi Chuan Xue Za Zhi 29: 300-305.

Lin IH, Ho ML, Chen HY, Lee HS, et al. (2012). Smoking, green tea consumption, genetic polymorphisms in the insulinlike growth factors and lung cancer risk. PLoS One 7: e30951.

MacDonald K, Porter GA, Guernsey DL, Zhao R, et al. (2009). A polymorphic variant of the insulin-like growth factor type I receptor gene modifies risk of obesity for esophageal adenocarcinoma. Cancer Epidemiol. 33: 37-40.

McElholm AR, McKnight AJ, Patterson CC, Johnston BT, et al. (2010). A population-based study of IGF axis polymorphisms and the esophageal inflammation, metaplasia, adenocarcinoma sequence. Gastroenterology 139: 204-212.

Moon JW, Chang YS, Ahn CW, Yoo KN, et al. (2006). Promoter -202 A/C polymorphism of insulin-like growth factor binding protein-3 gene and non-small cell lung cancer risk. Int. J. Cancer 118: 353-356.

Peters JL, Sutton AJ, Jones DR, Abrams KR, et al. (2006). Comparison of two methods to detect publication bias in metaanalysis. JAMA 295: 676-680.

Pollak MN, Schernhammer ES and Hankinson SE (2004). Insulin-like growth factors and neoplasia. Nat. Rev. Cancer 4: 505-518.

Pollak M (2008). Insulin and insulin-like growth factor signalling in neoplasia. Nat. Rev. Cancer 8: 915-928.

Ponder BA (2001). Cancer genetics. Nature 411: 336-341.

Rudd MF, Webb EL, Matakidou A, Sellick GS, et al. (2006). Variants in the GH-IGF axis confer susceptibility to lung cancer. Genome Res. 16: 693-701.

Serre D, Montpetit A, Pare G, Engert JC, et al. (2008). Correction of population stratification in large multi-ethnic association studies. PLoS One 3: e1382.

Stroup DF, Berlin JA, Morton SC, Olkin I, et al. (2000). Meta-analysis of observational studies in epidemiology: a proposal for reporting. Meta-analysis Of Observational Studies in Epidemiology (MOOSE) group. JAMA 283: 2008-2012.

Tabor HK, Risch NJ and Myers RM (2002). Candidate-gene approaches for studying complex genetic traits: practical considerations. Nat. Rev. Genet. 3: 391-397.

Vogelstein B and Kinzler KW (2004). Cancer genes and the pathways they control. Nat. Med. 10: 789-799.

Yi HK, Kim SY, Hwang PH, Kim CY, et al. (2005). Impact of PTEN on the expression of insulin-like growth factors (IGFs) and IGF-binding proteins in human gastric adenocarcinoma cells. Biochem. Biophys. Res. Commun. 330: 760-767.

Zintzaras E and Ioannidis JP (2005). HEGESMA: genome search meta-analysis and heterogeneity testing. Bioinformatics 21: $3672-3673$ 